\title{
Nuevas Tecnologías para el estudio sostenible de dimensiones físicas de centros educativos
}

\section{New Technologies for the sustainable study of physical dimensions of schools}

\author{
Santiago Rodríguez \\ Universidade de Vigo, España
}

\begin{abstract}
Resumen
Se expone aquí una experiencia llevada a cabo en un instituto público de Enseñanza Secundaria Obligatoria (ESO) de España orientada al estudio sostenible de sus dimensiones físicas mediante el uso de Nuevas Tecnologías de la Información y la Comunicación (TIC). El objetivo es fomentar valores y actitudes en pro del desarrollo sostenible en alumnado de $4^{\circ}$ de ESO a través de de un programa consistente en la realización de una auditoría ecológica a las dimensiones físicas del centro materializada a través del uso de TIC. La investigación realizada muestra que, tras la ejecución del proyecto, se consigue el objetivo perseguido.

Palabras clave: [ambiente escolar, entorno construido,
\end{abstract} Nuevas Tecnologías, Desarrollo Sostenible]

\begin{abstract}
Here we present an experience carried out in a public High School of Secondary Education in Spain aimed at the sustainable study of its physical dimensions through the use of New Information and Communication Technologies (ICT). The objective is to promote values and attitudes towards sustainable development in students of the $4^{\text {th }}$ year of Secondary Education through a program consisting of an ecological audit of the physical dimensions of the school through the use of ICT. The research shows that, after carrying out the project, the initial objective is achieved.

Keywords: [school environment, built environment, New Technologies, Sustainable Development]
\end{abstract}

\section{Introducción}

El desarrollo sostenible consiste en satisfacer las necesidades de las generaciones actuales sin poner en compromiso la capacidad de futuras generaciones de satisfacer sus propias necesidades (Keiner, 2006). En la actualidad, el carácter poco sostenible de nuestros modelos globales hace que la educación formal se erija como lugar imprescindible para fomentar un consumo responsable y mejorar el medio ambiente. Así, uno de los principales retos que la escuela ha de asumir es el de educar en la sostenibilidad (López, 2006). Cabe citar que la UNESCO ha reiterado el papel de la educación como instrumento imprescindible para "lograr el bienestar social, el desarrollo sostenible y la buena gobernanza" (UNESCO, 2014, p. 2).
Mención especial en relación al vínculo educación-desarrollo sostenible reciben las TIC, pues además de que ya no se pueden concebir los centros educativos actuales ajenos a ellas, resultando indispensables para los procesos de enseñanza-aprendizaje en pleno siglo XXI (Cebrián, Sánchez, Ruiz \& Palomo, 2009), también son imprescindibles para contribuir al ahorro de papel y energía, con los beneficios medioambientales derivados de ello (Hilty \& Aebischer, 2015).

Además, es indispensable subrayar la magnitud y repercusión de los procesos de enseñanza-aprendizaje involuntarios y no explícitos con base a las dimensiones físicas de los centros educativos. Esta idea bien se puede resumir con la siguiente cita: "un edificio escolar puede decir a los alumnos quiénes son y qué deben opinar sobre el mundo" (Grosvenor \& Burke, 2008). La idea de base es la concepción de los centros educativos como "herramientas culturales" cuyo diseño, construcción y uso hacen que ejerzan como elemento de mediación semiótica invisible en las prácticas que se llevan a cabo en ellos (Tse, Learoyd-Smith, Stables \& Daniels, 2015), dictando las reglas que conforman el discurso pedagógico y sus prácticas, y condicionando la formación de la concienciación de sus usuarios (Bernstein, 2000), ya sea para bien o para mal. El fundamento básico de estas afirmaciones se apoya en la teoría sociocultural de Vygotsky, donde el contexto sociocultural y el correspondiente complejo sistema de relaciones que éste supedita estructura las prácticas institucionales y condiciona sus efectos (Vygotsky, 1986). En definitiva, los centros educativos poseen una capacidad performativa que trasciende las acciones educativas explícitas que tienen lugar en ellos, mediante la interacción entre la cultura y el medio: "los espacios en los que (...) aprendemos pueden tener efectos profundos en cómo nos sentimos y cómo nos comportamos" (Watson, 2007, p. 260).

Por último cabe indicar que recientemente han surgido indicadores que permiten cuantificar de forma científicamente fidedigna el carácter sostenible o no sostenible de cualquier sistema u organización humana (Cagiao, 2009). Entre ellos destaca la huella de carbono, que es la conversión de todos los impactos de una organización a emisiones equivalentes de $\mathrm{CO} 2$ a la 
atmósfera. Una de las metodologías más consistentes para su cálculo es la metodología MC3 (Doménech, 2004). La estructura de la herramienta mediante la que se instrumentaliza esta metodología incluye todas las posibles categorías de consumo, además de ocupación de suelo y generación de residuos. Un punto de notable interés de esta metodología a efectos didácticos, es la manejabilidad de la herramienta mediante la que se instrumentaliza, pues se ha traducido a una hoja de cálculo de relativamente fácil ejecución.

Pues bien, este trabajo tiene por objeto fomentar valores y actitudes en pro del desarrollo sostenible en el alumnado de un grupo de $4^{\circ}$ de ESO de un centro educativo de titularidad pública a través de la puesta en práctica de un programa de intervención consistente en la realización por parte del alumnado de una auditoría ecológica mediante el uso de TIC a las características físicas del centro empleando la metodología MC3 (Doménech, 2004). Como hipótesis de partida se considera que la implicación del alumnado en el desarrollo sostenible de las dimensiones físicas del centro educativo mediante el uso de TIC contribuye al impulso de valores y actitudes en pro de la sostenibilidad.

\section{Metodología}

A efectos metodológicos, este proyecto constituye un estudio de caso único con diseño experimental de grupo pretest - postest (Campbell \& Stanley, 1978; Cohen \& Manion, 1990; Yin, 1994). Ahora bien, para dotar a la investigación de mayor rigor, también se ha considerado, además del grupo experimental, un grupo control.

De esta forma, como participantes directos en el estudio se considera:

Grupo experimental: alumnado de $4^{\circ}$ curso (grupo B) de ESO de la asignatura de Tecnología, ejerciendo de conductor y coordinador (para la intervención), el autor del presente trabajo en coordinación con el docente de la asignatura. El grupo está formado por 19 personas, 10 varones y 9 mujeres, de edades comprendidas entre los 15 y los 17 años. Siguiendo las recomendaciones del docente de Tecnología, este ha sido el grupo sobre el que se ha acometido la intervención.

Grupo control: grupo de características similares al anterior sobre el que no se ha realizado la intervención pero sí se les ha solicitado una redacción sobre su instituto ideal al mismo tiempo que la redacción post-test al grupo experimental. Se trata del alumnado de $4^{\circ}$ curso (grupo A) de ESO, formado por 20 personas, 8 varones y 12 mujeres, de edades comprendidas entre los 15 y los 16 años.

En todo caso, además del alumnado también es necesario destacar como participantes instrumentales en el estudio al docente de Tecnología, quien desde un primer momento se ha mostrado solícito para acometer el proyecto de intervención en sus clases, colaborando de forma activa para llevarlo a buen puerto (estando siempre presente durante su ejecución y resolviendo dudas al alumnado). Asimismo, cabe citar, análogamente, a una profesora de Lengua y Literatura gallegas, una mujer que ha colaborado activamente en el proyecto pues ha sido la encargada de solicitar en sus clases las producciones escritas al grupo experimental y control. Por último, también cabe citar la participación del equipo directivo, con el director y secretario del centro a la cabeza, encargados de facilitar la información de consumos y características del centro (superficies, gastos incurridos, cafetería, etc.) y, por supuesto, permitir la ejecución del proyecto.

Los pasos seguidos en el estudio han sido:

\section{Negociación del caso: autorizaciones y recopilación de información}

Teniendo en cuenta que el estudio acometido supone la 'intromisión de unas personas (investigadores) en la vida de otras (docentes, alumnado, (...), la negociación (...) es un compromiso en investigaciones en las que se emplean estudios de casos" (L.A.C.E., 1999, p. 11). Así, tras los primeros contactos informales con el docente de Tecnología, se procedió a celebrar una reunión formal con la dirección de la institución a fin de solicitar las autorizaciones para acometer el Proyecto y la información necesaria para ponerlo en práctica. En esta reunión participaron, además del autor de este Trabajo, el director del centro y el profesor de Tecnología. También se sumaría, a su finalización, el secretario, que fue el encargado de facilitar los datos de consumos del centro necesarios para ejecutar la auditoría ecológica. Las condiciones exigidas desde la dirección del centro para poner en práctica el Proyecto fueron:

a. Confidencialidad: garantía de que no se realizará a lo largo de la difusión del Trabajo ninguna referencia que pueda identificar al centro o a los participantes en el Proyecto.

b. Sistematización y brevedad de la intervención: minimización de la afección del desarrollo del Proyecto al devenir cotidiano del centro. Tras esta reunión, se mantuvo otra con la docente de Lengua y Literatura gallegas, en la que se le solicitó su colaboración para participar en el Proyecto y, una vez confirmada, se le facilitaron las instrucciones a fin de reunir los instrumentos cualitativos de investigación de este Trabajo.

\section{Redacción sobre el Instituto ideal realizada por el grupo experimental (pretest)}

Antes de la intervención, la docente de Lengua y Literatura gallegas, pide al grupo de $4^{\circ} \mathrm{B}$ de ESO del centro la realización de una redacción describiendo su instituto ideal, obteniendo, un primer bloque de información cualitativa. Las premisas dadas fueron:

c. Extensión máxima: una cara folio A4.

d. Anonimato (siguiendo las directrices del equipo directivo).

e. Medio de escritura: bolígrafo (no lápiz).

f. Idioma: gallego (por administrarse en clase de esta asignatura)

g. Tiempo máximo de elaboración: media hora. Se reúnen 18 producciones individuales.

\section{Fase de intervención en el aula con el grupo} experimental

Se realizó a lo largo de 4 sesiones de la asignatura de Tecnología, teniendo lugar en el aula de Informática del 
centro, que, además de disponer de 26 puestos informáticos para el alumnado, también cuenta con un ordenador para la docencia conectado a un cañón que proyecta las imágenes del ordenador del docente en una pantalla de gran tamaño, visible desde toda el aula. El conductor principal de la intervención ha sido el autor del presente Trabajo. Si bien, ha estado asistido en todo momento por el profesor de Tecnología. La intervención constó de las siguientes partes:

h. Conceptualización teórica (1 sesión): en esta sesión se impartió en el aula una introducción de los principales contenidos teóricos que constituyen la base fundamental de la concepción del desarrollo sostenible, claves para la posterior ejecución práctica en pro de la sostenibilidad y de su adecuado entendimiento e interpretación. Asimismo, se mostró al alumnado la herramienta de trabajo, comenzando a explicar su naturaleza y funcionamiento.

i. Ejecución de la auditoría ecológica por parte del alumnado mediante la herramienta de análisis de la metodología MC3 (3 sesiones): se dedicaron 3 sesiones a la ejecución de la auditoría ecológica. La primera de ellas consistió en mostrar en detalle el funcionamiento de la herramienta de trabajo y distribuir por grupos los datos a introducir. En la segunda sesión y parte de la tercera, el alumnado introdujo sus hallazgos en el instrumento, determinando los subtotales para cada categoría de la herramienta MC3 y comunicándolos al conductor de la intervención, que los introdujo en su propia hoja de cálculo a fin de compilar todos los resultados parciales y construir tablas y gráficos. Gran parte de la última sesión fue dedicada en formato de debate grupal a la discusión de los resultados obtenidos y extracción de conclusiones y eventuales propuestas de mejora por parte del alumnado. Con anterioridad a esta segunda subfase, el autor de este Trabajo adaptó la herramienta MC3 para facilitar su utilización por parte del alumnado y la introdujo en los ordenadores del aula.

Redacción sobre el Instituto ideal realizada por el grupo experimental (post-test) y por el grupo control

Pasados dos meses desde la finalización de la intervención, la docente de Lengua y Literatura gallegas, solicita, respectivamente, a los grupos experimental y control, la realización de una redacción describiendo su instituto ideal, obteniendo, así, el segundo y tercer bloque de información cualitativa. Las características de estos relatos responden a las mismas que las de los relatos pretest. Se reúnen 19 producciones individuales en el grupo experimental y 20 en el grupo control.

\section{Análisis de contenido de los relatos reunidos}

Siguiendo a Miles y Huberman (1994), se procedió a: codificar cada relato (se asignó un código alfanumérico a cada relato identificándolo individualmente); lectura general de todos los relatos y transcripción de los mismos (unificándose en formato único); reconocimiento y separación de las distintas unidades de información en cada relato; confección de una matriz de metadatos (hoja de cálculo) en la que se recoge para cada relato un etiquetado minucioso de las distintas unidades de información, con indicación de la frecuencia de aparición; confección de una matriz temática conceptual de metadatos donde se recoge para cada uno de los tres conjuntos de relatos una categorización del etiquetado del paso anterior según una metodología de taxonomía popular ("folk taxonomy", Miles \& Huberman, 1994, p. 133) basada en las clasificaciones de las dimensiones y subdimensiones del centro escolar de Cid-Sabucedo (2003); conclusiones e informe final.

\section{Resultados}

En primer lugar, y una vez realizada la auditoría ecológica por parte del alumnado, se observa que las categorías que peores resultados arrojan son las relativas a materiales no orgánicos (donde los materiales informáticos y tóner tienen el mayor peso), al consumo de electricidad y a los recursos forestales (empleo de materiales derivados del papel). Asimismo, también destaca el consumo relativo a la calefacción y, aunque en menor medida, el imputable a los residuos y vertidos del centro. Aunque sensiblemente inferiores a las anteriores categorías, el consumo de agua y el uso del suelo (meramente construido, sin zonas verdes) también tienen un impacto negativo. En el tramo final de la intervención, las propuestas del alumnado para mejorar la situación diagnosticada fueron la implantación de zonas verdes, la reducción, reutilización y reciclaje de papel y tóner, la separación de residuos mediante la instalación de contenedores específicos y la reducción del consumo eléctrico y de agua. Cabe decir que gran parte de estas propuestas están presentes en las características del Instituto ideal en los relatos post-test.

En segundo lugar, con respecto a las redacciones del grupo experimental pre-test, se han considerado 133 unidades de información distribuidas en 44 categorías, cada una de estas categorías se corresponde con una característica del Instituto ideal. La característica del Instituto ideal más citada con gran diferencia (18 ocasiones en 12 sujetos diferentes) es la que incide en un mayor uso de las Nuevas Tecnologías en las actividades del centro (fundamentalmente en las relativas a la docencia). Ahora bien, el alumnado subraya en general como justificación, no cuestiones medioambientales, sino el hecho del bienestar y confort personal que les produciría la sustitución de los recursos tradicionales de enseñanza-aprendizaje (libros, libretas, etc.) por recursos TIC a fin de aligerarles el peso de sus mochilas de forma significativa. Muy relacionado con esto, aunque con una frecuencia de aparición inferior a la mitad (7 ocasiones; 6 sujetos), se encuentra la demanda de poder usar los teléfonos móviles en el centro. También se insiste el mismo número de veces en que el centro educativo goce de un buen aspecto. Asimismo, es destacable cómo hasta 6 estudiantes diferentes demandan que las clases en su centro ideal comiencen más tarde por la mañana. El alumnado también insiste, aunque en menor medida (5 ó menos sujetos), en cuestiones relacionadas con las interrelaciones de centro, principalmente en aspectos vinculados a la docencia, comunicación y clima escolar, como pueden ser una menor exigencia y mayor cercanía del profesorado, clases de mayor utilidad vinculadas a la vida cotidiana, comportamientos respetuosos de toda la 
comunidad, etc. Análogamente, consideran que su centro ideal debería contar con una cafetería en condiciones, algo que llegan a citar hasta en 5 ocasiones 5 sujetos diferentes. Hay que destacar que la mitad de las características sugeridas por el alumnado para su Instituto ideal se encuadran en el subsistema estructural, perteneciendo la mayor parte de ellas a la estructuración de recursos materiales (y, específicamente, dentro de esta categoría a espacios y servicios del centro).

En tercer lugar, con respecto a las redacciones postest del grupo experimental, se han considerado 126 unidades de información distribuidas en 36 categorías. Ahora bien, es muy elocuente el hecho de que 7 de estas redacciones hayan sido escritas en folios de papel reciclado, algo que no sucede con ningún otro relato. Tal y como ocurría antes de la intervención, la característica del Instituto ideal más citada con gran diferencia (15 ocasiones en 10 sujetos diferentes) es la que incide en un mayor uso de las Nuevas Tecnologías en el centro. Sin embargo, el argumento que ahora esgrime el alumnado no sólo es el de su comodidad personal evitando el peso innecesario de las mochilas, sino que defienden el uso de las TIC como medida medioambiental para minimizar el consumo de recursos forestales generado por la utilización de materiales impresos. Además, es muy relevante cómo más de la mitad de los sujetos citan cuestiones relativas a la estructuración de recursos materiales del centro de índole estrictamente medioambiental y carácter sostenible: la reducción del consumo eléctrico y del consumo en calefacción y la separación de residuos. Así, las características del Instituto ideal que más cita el alumnado en los relatos post-test están directamente relacionadas con aspectos relativos a la sostenibilidad de las dimensiones físicas del centro, que es la diferencia más destacable con los relatos realizados antes de la intervención. Además, en las redacciones post-test aparecen nuevas características del Instituto ideal que no aparecían en los relatos reunidos previos a la intervención: el uso de bicicletas, ubicar plantas en las aulas, reducir papel empleando folios usados, reducir tóner, localizar un contenedor para tóner, usar papel reciclado, reducir el consumo de agua, implantar un transporte escolar colectivo, uso de energías renovables, etc. En este caso, tres cuartas partes de las características sugeridas por el alumnado para su Instituto ideal pertenecen al subsistema estructural, de las cuales la mayoría hacen referencia a aspectos relacionados con la estructuración de recursos materiales del centro.

En cuarto lugar, en los relatos del grupo control se consideran 188 unidades de información distribuidas en 43 categorías. A diferencia de los relatos del grupo experimental, las características del Instituto ideal más nombradas en el grupo control tienen que ver con aspectos docentes y relacionales. Así, en este caso, tres cuartas partes del total de unidades de información encontradas en los relatos del grupo control se reparten de manera bastante uniforme entre el subsistema estructural y relacional, destacando el número de características del centro ideal vinculadas a aspectos relacionales tales como los estrictamente docentes o el clima, la comunicación, la motivación o la participación.
Por último, y ya específicamente referido a aspectos vinculados con la sostenibilidad del centro, cabe decir que en las redacciones pretest del grupo experimental, sólo 4 categorías que comprenden un total de 8 unidades de información, se relacionan directamente con aspectos sostenibles, mientras que en el grupo de relatos experimental postest se detectan 79 unidades de información distribuidas en 16 categorías con relación directa con aspectos asociados con la sostenibilidad del centro. Así, en las redacciones post-test del grupo experimental se han considerado cerca de dos tercios de unidades de información relacionadas con la sostenibilidad sobre el total $(62,7 \%)$, suponiendo un salto notable con respecto a la situación previa a la intervención. Además, las redacciones del grupo experimental pretest y del grupo control son bastante semejantes en este aspecto, considerándose, respectivamente, un $6 \%$ y un $0,5 \%$ de unidades de información "sostenibles" en relación a los totales considerados. Además, a diferencia de lo que ocurre en las redacciones del grupo experimental pretest ( 8 sujetos sobre un total de 18) y del grupo control (1 sujeto sobre un total de 20), el $100 \%$ del alumnado del grupo experimental cita tras la intervención como característica de su instituto ideal alguna cuestión que lo haga más sostenible.

Además, aun sin existir una correspondencia directa entre los aspectos del centro ideal de los que se habla en los relatos post-test y el diagnóstico realizado a través de la metodología MC3 durante la intervención, sí que se observa una cierta correlación entre el número de unidades de información detectados para cada categoría MC3 y los resultados de la auditoría ecológica en cada categoría, lo que refuerza la repercusión que ha tenido la ejecución del Proyecto con el alumnado participante.

\section{Conclusiones}

A la vista de los resultados obtenidos, se ha podido constatar cómo a través de una intervención que se ha apoyado exclusivamente en TIC, se ha alcanzado el objetivo previsto verificando la hipótesis de partida. De esta manera, se ha contribuido a hacer germinar en el alumnado participante la semilla de la sostenibilidad, de tal modo que, además de hacerlos conscientes de su importancia, también practiquen eventualmente en su vida cotidiana actitudes sostenibles.

Una de las cuestiones que se consideran fundamentales a efectos de haber obtenido éxito con la intervención es el protagonismo del alumnado, pues han sido los propios estudiantes los encargados de llevar a cabo la auditoría ecológica a su centro durante la fase de intervención. En este sentido, la literatura existente informa de las positivas repercusiones educativas de implicar a los miembros de una misma comunidad de forma práctica y directa dentro del contexto propio durante la ejecución de cualquier acción de cara al fomento de una positiva concienciación medioambiental, en el sentido de consolidar su sentimiento de pertenencia a ese entorno e involucrarse en su mantenimiento, visibilizando la capacidad performativa de ese contexto, en este caso, las dimensiones físicas del centro educativo (Grosvenor \& 
Burke, 2008; Pata \& Metsalu, 2008; Echeita \& Navarro, 2014; Tse, Learoyd-Smith, Stables \& Daniels, 2015; Bernstein, 2000; Watson, 2007).

Además, es necesario subrayar su constante interés a lo largo de toda la fase de intervención, pues el alumnado se ha mostrado en todo momento altamente motivado y con una gran disposición al trabajo, algo que acaso tenga mucho que ver con el uso de las TIC a lo largo de la puesta en práctica de la experiencia, pues las TIC suponen un valioso apoyo para promover, motivar y activar el aprendizaje (Muntaner, 2005), teniendo un gran efecto facilitador en él (Alcántara, 2009). A esto hay que añadir la aplicabilidad que presentan en la vida cotidiana (Winocor, 2007), que en el caso de este estudio, al estar analizando su propio centro, es máxima. Por otra parte, y tal y como afirman autores como Gallego y Alonso (1999), las TIC también han servido para captar fácilmente el interés y la motivación del alumnado, mostrando su versatilidad, gran velocidad de penetración y aplicabilidad. Por último, este estudio, que en la parte donde ha intervenido el alumnado se ha desarrollado de forma colaborativa, también resulta elocuente para poner de manifiesto la utilidad de herramientas tecnológicas como las empleadas para promover un aprendizaje activo y colaborativo entre estudiantes (Padilla-Melendez, Garrido-Moreno \& Aguila-Obra, 2008; Martins \& Kellermanns 2004).

\section{Referencias}

Alcántara, M. D. (2009). Importancia de las TIC para la educación. Innovación y experiencias educativas, 15 , 200-220.

Bernstein, B. (2000). Pedagogy, symbolic control and identity. Theory, research, critique. Lanham: Rowman \& Littlefield.

Cagiao, J. (Ed.) (2009). Ingeniería civil para un mundo sostenible. A Coruña: Fundación Ingeniería Civil de Galicia.

Campbell, D. \& Stanley, J. (1978). Diseños experimentales y cuasiexperimentales en investigación social. Buenos Aires: Amorrortu Editores.

Cebrián, M., Sánchez, J., Ruiz, J. \& Palomo, R. (2009). El impacto de las TIC en los centros educativos: ejemplos de buenas prácticas. Barcelona: Síntesis.

Cid-Sabucedo, A. (2003). La escuela como organización. En A. Blanco (Coord.), Didáctica General y Formación del Profesorado (pp. 99-138). Vigo: Servicio de Publicaciones de la Universidad de Vigo.

Cohen, L. \& Manion, L. (1990). Métodos de investigación educativa. Madrid: La Muralla.

Doménech, J. L. (2004). Huella ecológica y desarrollo sostenible. Madrid: AENOR Ediciones.

Echeita, G. \& Navarro, D. (2014). Educación inclusiva y desarrollo sostenible. Una llamada urgente a pensarlas juntas. Edetania, 46, 141-161.

Frey, H. \& Yaneske, P. (2007). Visions of sustainability. New York: Taylor \& Francis.

Gallego, D. J. \& Alonso, C. M. (Eds.) (1999). Multimedia en la web. Madrid: Dykinson.

Grosvenor, I. \& Burke, C. (2008). School. London: Reaktion Books.
Hilty, L. M. \& Aebischer, B. (Eds.) (2015). ICT Innovations for Sustainability. Zurich: Springer

Keiner, M. (2006). The future of sustainability. Dordrecht: Springer.

L. A. C. E. (1999). Introducción al estudio de caso en Educación. Universidad de Cádiz: Laboratorio para el Análisis del Cambio Educativo, Facultad de Ciencias de la Educación. Recuperado de https://goo.gl/FpHPjH

López, F. (Coord.) (2006). La sostenibilidad, un compromiso de la escuela. Barcelona: Graó.

Martins, L. L. \& Kellermanns, F. W. (2004). A model of business school students' acceptance of a web-based course management system. Academy of Management Learning and Education, 3(1), 7-26.

Miles, M. B. \& Huberman, A. M. (1994). Qualitative Data Analysis. Thousand Oaks: Sage Publications.

Muntaner, J. (2005). Los retos educativos del siglo XXI. Comunicación y Pedagogía, 204, 19-24.

Padilla-Meléndez, A., Garrido-Moreno, A. \& Aguila-Obra, A. R. D. (2008). Factors affecting e-collaboration technology use among management students. Computers \& Education, 51(2), 609-623.

Pata, K. \& Metsalu, E. (2008). Conceptualizing Awareness in Environmental Education: An Example of Knowing about Air-Related Problems. Science Education International, 19(1) 41-64.

Rees, W. \& Wackernagel, M. (2001). Nuestra Huella Ecológica: Reduciendo el Impacto Humano sobre la Tierra. Santiago de Chile: LOM.

Tse, H. M., Learoyd-Smith, A., Stables A. \& Daniels, H. (2015). Continuity and conflict in school design: a case study from Building Schools for the Future. Intelligent Buildings International, 7(2-3), 64-82.

UNESCO, Organización de las Naciones Unidas para la Educación, la Ciencia y la Cultura (2014). Documento de posición sobre la educación después de 2015. París: Autor. Recuperado de https://goo.gl/ckDNXF

Vygotsky, L. S. (1986). Thought and Language. Cambridge: The Massachuttes Institute of Technology.

Watson, L. (2007). Building the future of learning. European Journal of Education, 42(2), 255-263.

Winocor, R. (2007). Nuevas tecnología y usuarios. La apropiación de las TIC en la vida cotidiana. Telos. Cuadernos de Comunicación e Innovación, 73, 109-117.

Yin, R. K. (1994). Case Study Research. Design and Methods. London: Sage Publications. 